\title{
New grids of stellar models including tidal-evolution constants up to carbon burning
}

\author{
IV. From 0.8 to $125 M_{\odot}$ : high metallicities $(Z=0.04-0.10)^{\star, \star \star}$
}

\begin{abstract}
A. Claret
Instituto de Astrofísica de Andalucía, CSIC, Apartado 3004, 18080 Granada, Spain

e-mail: claret@iaa.es

Received 26 October 2006 / Accepted 28 January 2007

\section{ABSTRACT}

Aims. The aim of the present grid of stellar models is to complete our previous calculations and provide a tool for investigating the astrophysical properties of eclipsing binaries, stellar clusters, galactic bulges, and elliptical galaxies with a high metal content. Methods. To explore the applicability of high metallic models, we have computed three grids: $(X, Z)=(0.64,0.04),(0.58,0.06)$, and $(0.46,0.10)$. For all these grids, we adopted an enrichment law $\Delta Y / \Delta Z=2.0$, as in our previous papers on this subject. The input physics is almost the same as adopted in our earlier work except for some numerical details, recent measurement of the rate for the reaction ${ }^{14} \mathrm{~N}(p, \gamma){ }^{15} \mathrm{O}$, and the recent mass-loss rate for the Wolf-Rayet stages.

Results. Two high-metallicity clusters, NGC 6253 and NGC 6791, were used to test the present calculations with very satisfactory results. On the other hand, as this series of grids was mainly designed to investigate the tidal evolution of close binaries, we analyse the present status of circularization times in both clusters and isolated binaries. The present models ( 90 tables) can be retrieved from the CDS via anonymous ftp.
\end{abstract}

Key words. binaries: eclipsing - stars: evolution - stars: abundances - stars: rotation

\section{Introduction}

Eclipsing binary stars that show high metal content are not all that common. In the few detected cases, the metal content was detected due to spurious comparison with old stellar models based on old opacities and nuclear reaction rates. In spite of this, there is observational evidence for high metallic stars in clusters and in at least two double-lined eclipsing binaries. The two conspicuous cases of metal-rich clusters are NGC 6791 (Carraro et al. 2006) and NGC 6253 (Twarog et al. 2003). NGC 6791 is an old, nearby cluster with high metallicity. Depending on the adopted stellar models, their ages are between 8 and 12 Gyr. The metallicity of this cluster is still imprecise, but recently, Gratton et al. (2006) obtained high-resolution spectrography of red clump stars. Using a spectrum synthesis analysis, they were able to derive $[\mathrm{Fe} / \mathrm{H}]=+0.47 \pm 0.04$. On the other hand, Twarog et al. (2003) obtained CCD photometry in the Strömgren system $+\mathrm{Ca} \mathrm{H} \beta$ for NGC 6253. By using the $\delta m_{1}$ and $\delta h k$ indices, they found $[\mathrm{Fe} / \mathrm{H}]$ ranging from +0.7 up to +0.9 . The system with highest metallicity of double-lined eclipsing binaries, as far as we known, is WW Aur, which has been studied in detail by Southworth et al. (2005). The average accuracies of the radii and masses are smaller than $1 \%$. The observations can be fitted only for metal-rich models $(Z=0.06)$, although there is no clear connection with the metallic lines present in the system.

* Additional data are available on CD ROMs upon request.

$\star \star$ Model data (90 tables) is only available in electronic form at the CDS via anonymous ftp to

cdsarc.u-strasbg.fr $(130.79 .128 .5)$ or via

http://cdsweb.u-strasbg.fr/cgi-bin/qcat?J/A+A/467/1389
Metal-rich stars are expected to also be present in galactic bulges and elliptical galaxies, with $Z$ as high as 5 times the solar value (e.g. Moehler \& Sweigart 2006; Sadler 1992). Higher metallicities (10-15 times $Z_{\odot}$ ) have been assignated to quasars (Korista et al. 1996; Baldwin et al. 2003) though the inferred values are somewhat uncertain. The aim of the present grid of metal-rich stellar models is to complete our previous calculations and provide a tool for investigating the astrophysical properties of eclipsing binaries, stellar clusters, galactic bulges, and elliptical galaxies with high metallicity.

The present grids, together with those previously published (Claret 2004, 2005a, 2006), enable us to analyse their impact on the tidal evolution of close binaries whether located in clusters or isolated. We study the effects of changing the radius, mass, and depth of the convective layer on the circularization times in order to evaluate the impact of theoretical uncertainties. Even taking such uncertainties into account we show that the tidalbreaking and the radiative damping mechanisms are not efficient enough to match the observed levels of circularization.

\section{Characteristics of the models}

In order to explore the applicability of high metallic models, we have computed three grids: $(X, Z)=(0.64,0.04),(0.58,0.06)$, and $(0.46,0.10)$. For all these grids, we adopted an enrichment law $\Delta Y / \Delta Z=2.0$, as in our previous papers on this subject. The input physics is almost the same as adopted in Claret (2004). However, some updates were implemented concerning nuclear reaction rates (Runkle 2003; Formicola et al. 2004), numerical accuracy (e.g. the size of the triangle used to define an envelope in the HR diagram that is important for avoiding numerical 


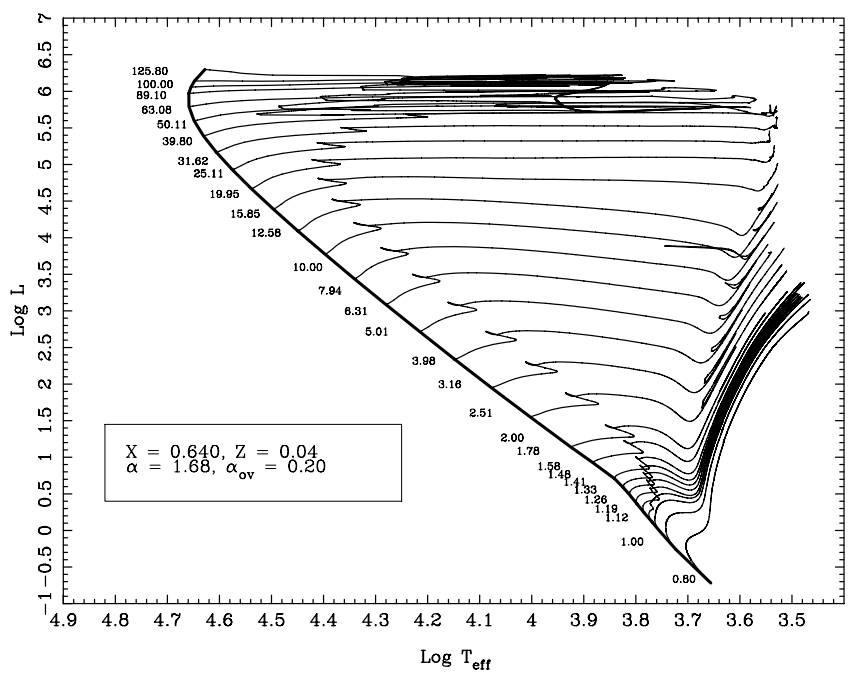

Fig. 1. The theoretical HR diagram for $Z=0.04$. The numbers below the tracks indicate the initial stellar masses in solar units.

oscillations in $x_{\mathrm{bf}}$ - see Fig. 9) and, mass loss rates for the WolfRayet stages, in massive stars. For the latter we have adopted the recent formalism by Nugis \& Lamers (2000), which takes the dependence on metallicity and luminosity into account. We do not describe the adopted input physics here, but for a quick reference we give a summary in Table 1 of the main characteristics of the present grids.

For completeness, we briefly discuss the mass loss of massive stars. As pointed out by Mowlavi et al. (1998), the criterion for defining a Wolf-Rayet star may be different for the one adopted for lower metallicities. Due to the large uncertainties involved, we adopt the same criterion here as was considered in our previous papers. In contrast to Mowlavi et al. (1998), who stop the calculations at $60 M_{\odot}$, we computed models up to $125 M_{\odot}$, always keeping in mind the large inaccuracy of the mass-loss rates in this mass range. The corresponding HR diagrams for the three grids can be seen in Figs. 1-3 for $(X, Z)=(0.64,0.04),(0.58,0.06)$, and $(0.46,0.10)$, respectively. One of the showiest characteristic of the massive stars in these figures is that, due to their lower initial hydrogen content, they reach the Wolf-Rayet stage earlier, still during the mainsequence. Mowlavi et al. (1998) find that the models that are more massive than $60 M_{\odot}$ lose almost their initial mass during the main-sequence at $Z=0.10$. Our results do not completly agree with this. For example, a model with an initial mass of $125 M_{\odot}(Z=0.10)$ achieves the end of the main-sequence with about $30 M_{\odot}$. Such a discrepancy is probably due to the different adopted mass-loss rates.

Another interesting feature of the high metallic models is that they are hotter and more luminous than their metal-poor counterparts, as already pointed out by Mowlavi et al. (1998), who analyse the case of a $3 M_{\odot}$ model. A simple homology approximation can be used to illustrate this feature: the luminosity depends on the mean molecular weight as $\mu^{a}$, where $a$ is greater than zero. Considering, for example, $\mu$ for $(X, Z)=(0.64,0.04)$ and $(0.46,0.10)$ for a fixed mass, a decrease in the hydrogen content and an increase in $Z$ implies a heavier mean molecular weight, hence an increase in luminosity. This effect can be inspected in Fig. 4 for the case of a $10 M_{\odot}$ model where the high luminosities and effective temperatures of $(X, Z)=(0.46,0.10)$ models can be easily noted if compared with the less metallic ones. On the other hand, lifetimes of hydrogen-burning phase

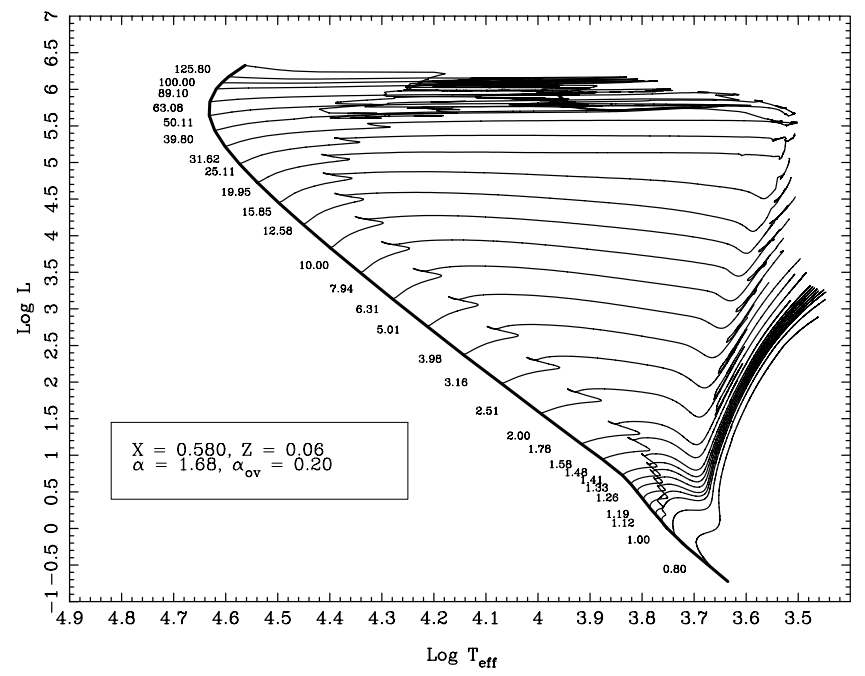

Fig. 2. The theoretical HR diagram for $Z=0.06$. Same remarks as in Fig. 1.

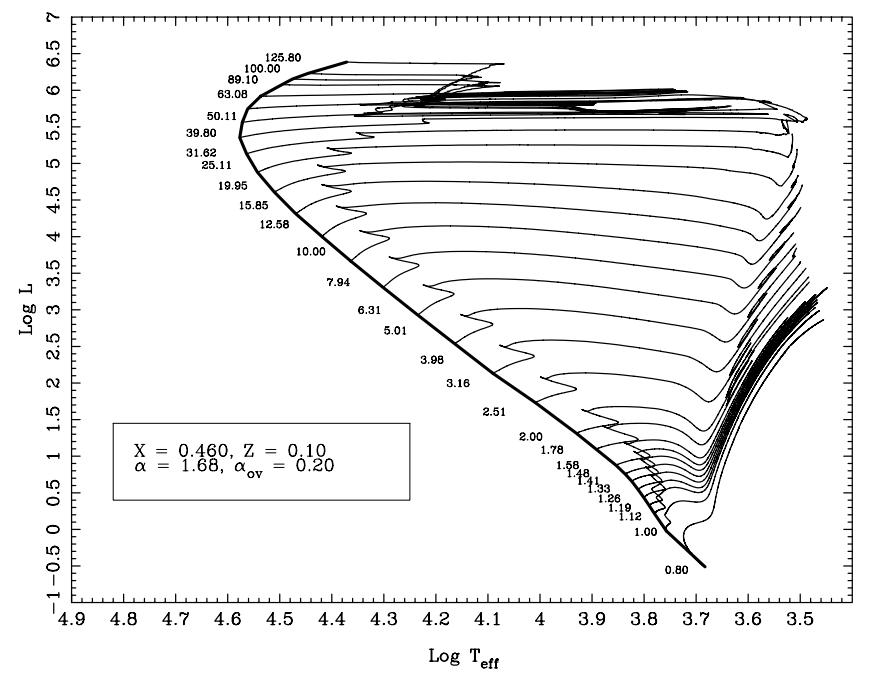

Fig. 3. The theoretical HR diagram for $Z=0.10$. Same remarks as in Fig. 1.

are expected to be shorter in more metallic models due to their lower hydrogen content. Their higher luminosities and effective temperatures also contribute to a decrease in the lifetimes. For example, we have found that the lifetime ratio (almost independent on mass) of the $(0.64,0.04)$ set and the $(0.46,0.10)$ one is around 2.3 (Fig. 5). In the case of core helium-burning, the situation is similar but the ratio of lifetimes between both sets is reduced to about 1.8 . For models with masses higher than $\approx 40 M_{\odot}$, the lifetime of core helium burning is almost independent of the mass.

\section{Internal structure}

As in the previous papers of this series, some parameters related to tidal evolution of close binaries systems are also computed. In this way, the harmonics $k_{2}, k_{3}$, and $k_{4}$ are available for testing the mass distribution of the stars showing apsidal-motion. Still concerning tidal evolution, the synchronization/circularization times can also be computed (and compared with the derived ages) by using the depth of the convective envelope, the tidal constant $E_{2}$, etc., which are provided for each model. 


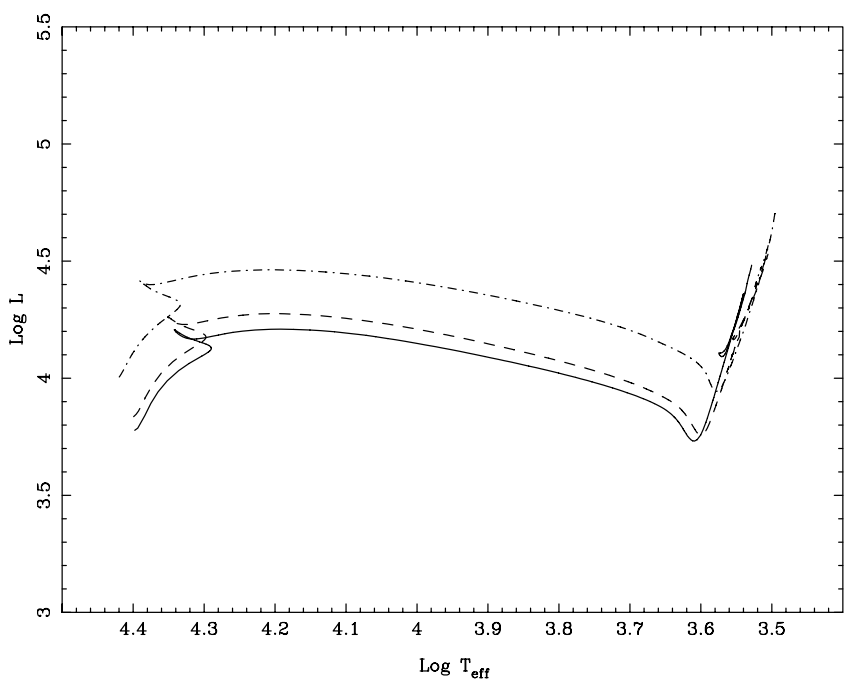

Fig. 4. The comparative HR diagram for an initial mass of $10 M_{\odot}$ at different metallicities. The continuous line represents the models with $(X, Z)=(0.64,0.04)$, dashed line denotes $(0.58,0.06)$, and dasheddotted line indicates $(0.46,0.10)$.

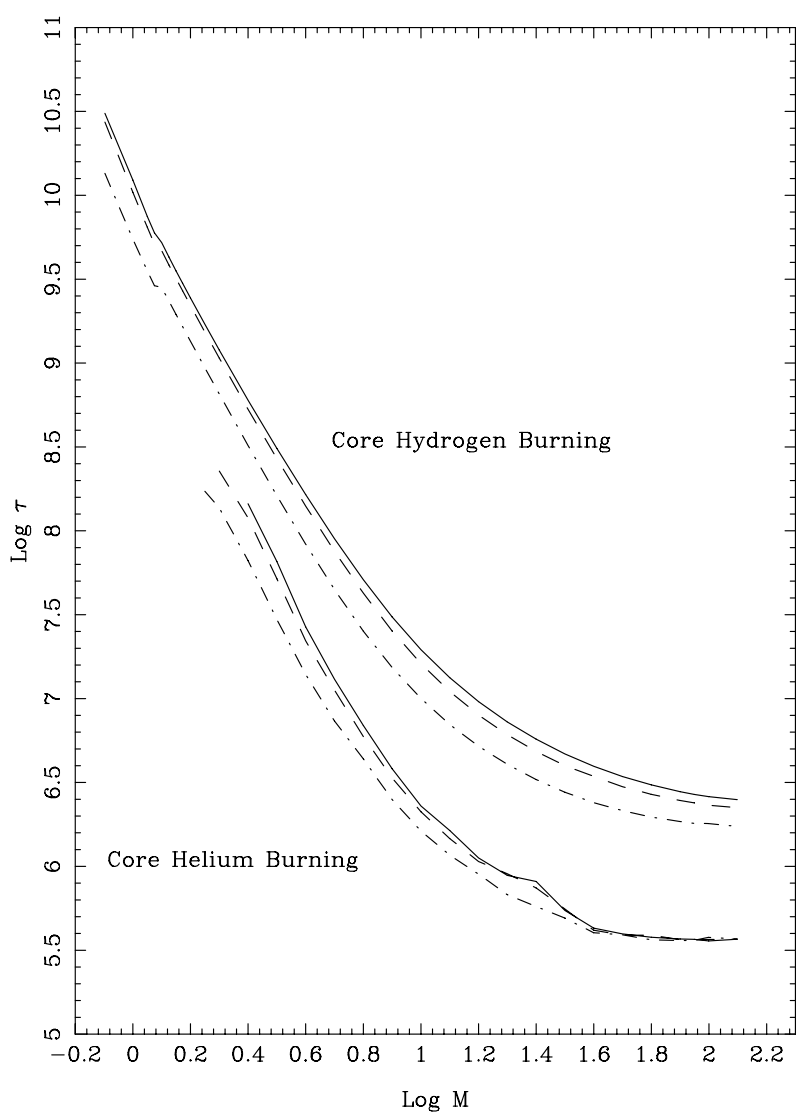

Fig. 5. The lifetimes for the hydrogen and helium-burning. Lines as in Fig. 4.

It is interesting to investigate the effects of metallicity on the harmonic $k_{2}$. We represent the apsidal-motion constant in Fig. 6 as a function of logarithm of mass (in solar units) for homogeneous models. For more massive models $(\log m \geq 1.2)$, the differences are very large, as we consider more metallic models. Indeed, the differences can reach 3 orders of magnitude. The same happens with the radius of gyration that describes the moment of inertia. Both features show that the metal-rich

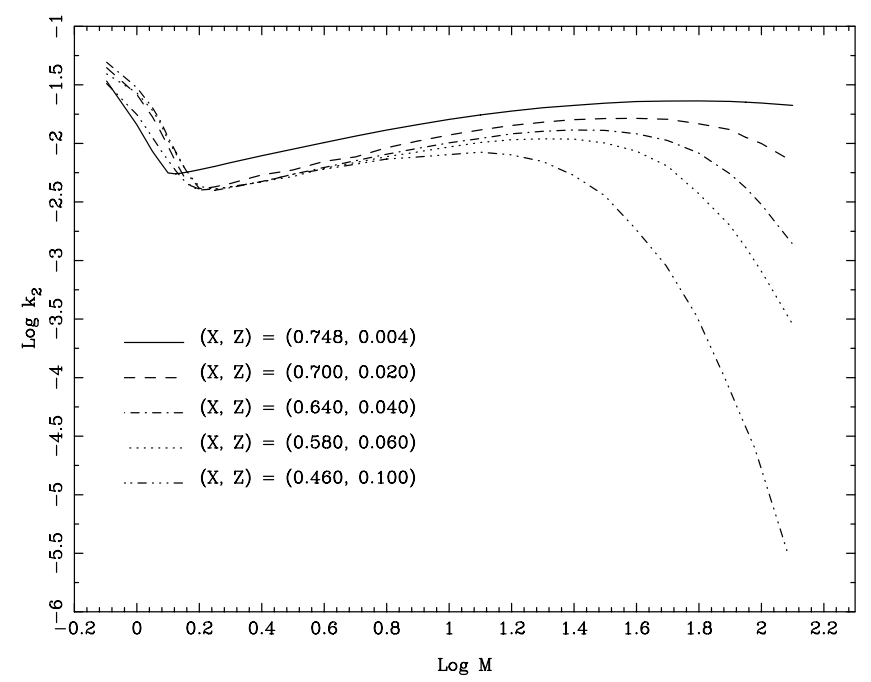

Fig. 6. Effects of metallicity on the harmonic $k_{2}$.

models present a more centrally concentrated mass distribution than models with the solar chemical composition, mainly for the more massive ones. The convective core size increases with the metal content, contributing to an increase in the degree of mass concentration. Of course, the hydrogen/helium content also plays an important role since it is not fixed for each grid. However, the main source of the strong mass concentration of more metallic models is the radius. Again, using a simple homology relation, it is not difficult to illustrate the role of the mean molecular weight on the harmonic $k_{2}$. For a fixed mass, the radius depends on the mean molecular weight as $\mu^{b}$, where $b$ is greater than zero. The apsidal-motion constant depends, in a first approach, on the mean density. Therefore, $k_{2}$ depends on $\mu^{-3 b}$, and using the same set as above, we finally find that the $(0.46,0.10)$ set shows a more centrally concentrated mass distribution. For details on the implications of this series of grids in circularization times, see Sect. 5.

Still concerning the internal structure but also connected with the stellar atmospheres, we present calculations of how much a star is distorted by rotation and/or tides. Rapid rotators and distorted stars (such as the components of close binaries systems) show a change in their surface brightness distribution. The actual accuracy of some measurements (see for example, Rattenbury et al. 2005) demands more precise calculations of flux distribution over the surface of rapidly rotating or tidally distorted stars. The flux depends on the local gravity through the gravity-darkening exponent $\beta_{1}$, which in turn depends on the predominant mechanism of transport of energy. The numerical method of deriving $\beta_{1}$ is based on the triangle strategy (Claret 1998, 2000), and the gravity-darkening exponents are available for each point of the evolutionary tracks. Therefore, the flux distribution can be computed for isolated rotating stars to investigate, for example, the influence of rotation on isochrones or for distorted components - by rotation and tides - of close eclipsing binaries.

\section{Some observational tests}

As already mentioned in the introduction, binary stars with a metal-rich content are not common. However, as pointed out, the clusters NGC 6253 and NGC 6791 seem to present metallicities well above the solar value. Therefore, we think that such clusters are good tests of the present theoretical models. 
Table 1. Summary of the input physics of the models.

\begin{tabular}{ll}
\hline \hline Mass range & $0.8-125 M_{\odot}$ \\
Chemical composition & $(X, Z)=(0.640,0.04),(0.580,0.06),(0.460,0.10)$ \\
Mixing-length parameter $\alpha$ & 1.68 \\
Core overshooting $\alpha_{\mathrm{ov}}$ & 0.20 \\
Opacities (high temperatures) & Iglesias \& Rogers (1996) \\
Opacities (low temperatures) & Alexander \& Fergunson (1994) \\
Neutrinos & Itoh et al. (1989) \\
Mass loss rate (Wolf-Rayet) & Nugis \& Lamers et al. (2000) \\
\hline
\end{tabular}

NGC 6253 was investigated by Twarog et al. (2003) using the $u v b y \mathrm{CaH}_{\beta}$ system. In spite of the high contamination of field stars - the cluster is located toward the galactic centre - they were able to obtain $[\mathrm{Fe} / \mathrm{H}]$ between +0.7 and +0.9 . The indices $\delta m_{1}$ and $\delta h k$ were used to estimate these metallicities. The inferred ages are 2.5-3.5 Gyr and $(m-M)=11.6$ and 12, respectively. In Fig. 7a we present our fit for NGC 6253 for the Strömgren $(b-y)$ index. The distance modulus is 12.0 , while we adopted $E(b-y)=0.20$, as given by Twarog et al. (2003). The isochrones were computed for $(X, Z)=(0.64,0.04)$. The fit can be considered as good and consistent with previous results, mainly taking the contamination for field stars into account.

Recently, Carraro et al. (2006) studied NGC 6791 and used isochrones for $Z=0.046$ and $Z=0.04$ to derive the distance modulus and age. The derived age from that analysis is 8 Gyears for a distance of $4.3 \mathrm{kpc}$. On the other hand, Gratton et al. (2006) observed four red clump stars to derive the metallicity of NGC 6791: $[\mathrm{Fe} / \mathrm{H}]=+0.47 \pm 0.04$, which is around $20 \%$ higher than the value inferred by Carraro et al. (2006). We adopted $(X, Z)=(0.58,0.06)$ as representative of this cluster. The result can be seen in Fig. $7 \mathrm{~b}$ for the Johnson $(B-V)$ index. The derived age and distance modulus agree with the values inferred by Carraro et al. (2006).

Another test, already done by Southworth et al. (2005), refers to WW Aur. This metallic-lined system has components with masses of $1.964 \pm 0.007$ and $1.814 \pm 0.007 M_{\odot}$ and radii of $1.927 \pm 0.011$ and $1.841 \pm 0.011 R_{\odot}$ for the primary and secondary, respectively. To obtain the effective temperatures, the Hipparcos parallax and ultraviolet, optical, and infrared fluxes were used. The absolute dimensions of both components of WW Aur seem to be matched only for high metallic models, say, $Z$ of the order of 0.06. As pointed out by Southworth et al. (2005), a natural question arises whether the metallic lines of WW Aur affect the radii or if it is just a surface phenomenon. They conclude that there is no reason to connect the changes in the radii to the Am phenomenon. Although an acceptable agreement with theoretical models was achieved, the current tidal theories are not able to explain the observed zero eccentricity of WW Aur. Finally, they conclude that the high metallicity required to match both components of WW Aur cannot be attributed to differences among the evolutionary codes and/or input physics.

\section{Circularization times in close binary systems}

Tidal interactions tend to circularize the orbits of close binaries. However, the involved mechanisms and their efficiency are not understood well yet. As an additional piece of the puzzle, the investigation of the eccentricity-period distribution in late-type binaries belonging to clusters provides an evolutionary clock (Mayor \& Mermilliod 1984): it was observed that such binary stars present a critical period (the cut-off period $P_{\text {cut-off }}$ ) above
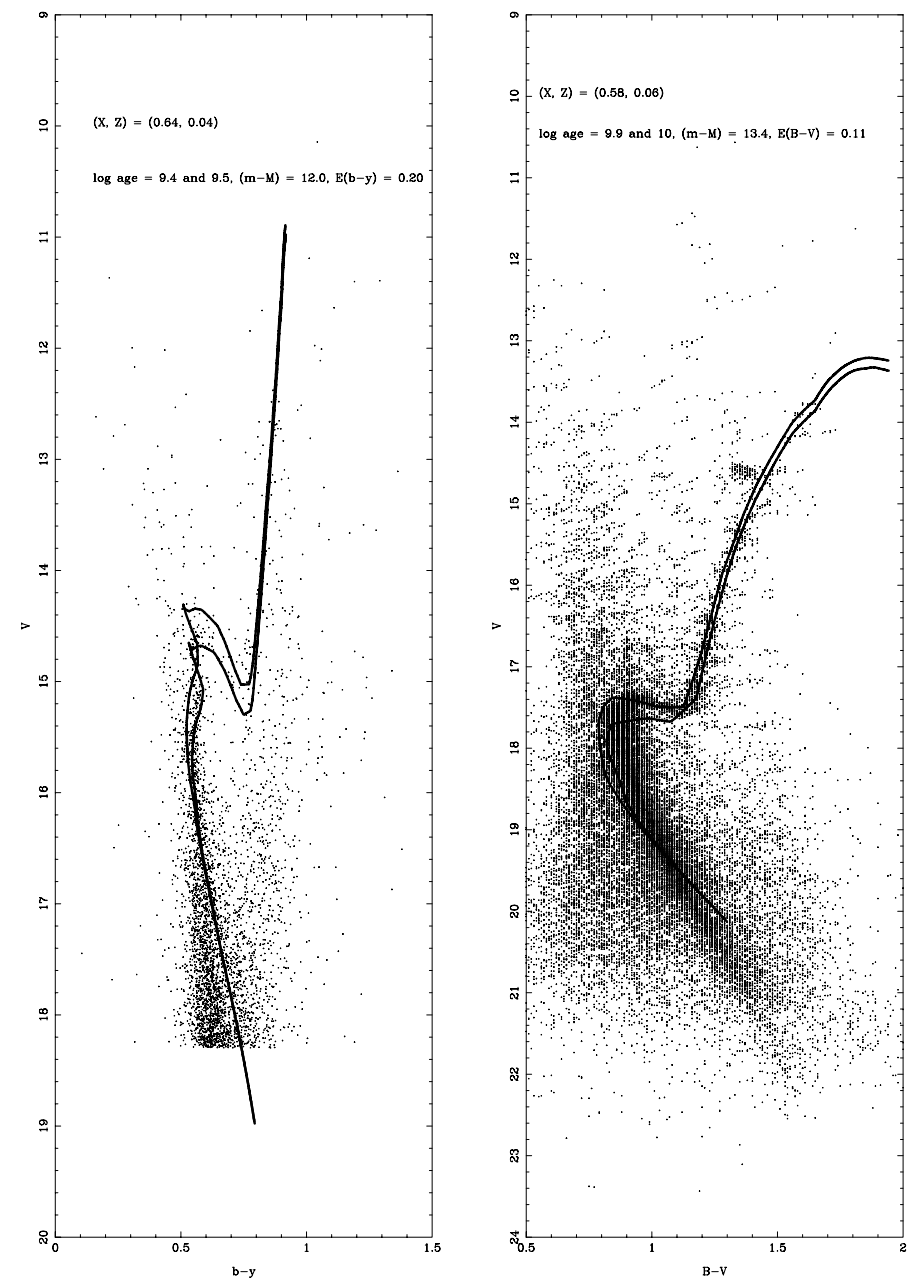

Fig. 7. Left Isochrones for NGC 6253. Log age $=9.4$ and 9.5. The adopted chemical composition was $(X, Z)=(0.64,0.04)$. The shift in the isochrones was $E(b-y)=0.20$ and $(m-M)=12.0$. Right Isochrones for NGC 6791. Log age $=9.9$ and 10.0. The adopted chemical composition was $(X, Z)=(0.58,0.06)$. The shift in the isochrones was $E(B-V)=0.11$ and $(m-M)=13.4$.

which the orbits are still eccentric. A relationship was then established between the age of the cluster and the corresponding $P_{\text {cut-off. The efficiency of the tidal-torque mechanism does not }}$ seem enough to fit such observations. However, there are important uncertainties in the comparison procedure that are often neglected. In this section we address some questions on the operational method, uncertainties, and definitions involving tidal evolution of binary systems located in clusters.

Two mechanisms are often invoked to explain the levels of circularization in close binary stars: the tidal-torque and the so-called hydrodynamical mechanism. In the tidal-torque 
mechanism, a tidal bulge appears due to the mutual interaction of the gravitational fields. If the stars are not synchronized, this bulge is not aligned and a delay in this perturbed region gives rise to a torque that tends to circularize the orbit. Zahn (1977, 1989) identified the radiative damping and the turbulent friction for stars with convective core or convective envelopes, as the respective agents. The second mechanism (Tassoul 1995) is characterised by large-scale hydrodynamical currents caused by the distortions due to tides. This mechanism requires a pre-calibration of the parameter $\gamma$ (Claret et al. 2005), and it is much more efficient than the tidal-torque one. However, Rieutord (1992) severely criticised this mechanism, which was later refuted by Tassoul \& Tassoul (1996).

\subsection{Uncertainties in the levels of circularization in clusters}

The investigations of circularization levels in binary stars belonging to clusters allow us to handle a large coeval sample but simultaneously introduce some uncertainties into the process of comparison with the theoretical predictions. In contrast to double-lined binaries, the absolute dimensions of binaries belonging to clusters are not well-determined. As an immediate consequence, their age determination is more problematic. Here we address some additional questions concerning the uncertainties involved in the process of comparing age versus $P_{\text {cut-off }}$. They are not necessarily in order of importance. In some of them, we have introduced some improvements, such as evaluating the theoretical error bars for the first time.

1) A similar set of stellar models must be used simultaneously to infer the age of clusters and to compute the critical times of circularization to avoid spurious differences due to the input physics of the interior models. This was done here by using our recent grids of stellar models. Although this can be considered as a definitive improvement, recall that it does not eliminate the problem of determinating the age of clusters (e.g. the reddening) and the intrinsic uncertainties of the stellar models (core overshooting, mixing induced by rotation, opacities, nuclear reaction rates, etc.). As an example of such uncertainties, we can quote the recent measurement of the nuclear reaction ${ }^{14} \mathrm{~N}(\mathrm{p}, \gamma){ }^{15} \mathrm{O}$ (Runkle 2003; Formicola et al. 2004), which reduces the usual rate to around $50 \%$. The influence of this new nuclear rate on determinating of age of the young clusters is obvious. For older clusters, the difference can reach about $10 \%$.

The effects of stellar rotation are not frequently considered in isochrone calculations (Meynet 1998). If one calls that rotation changes, in some cases drastically, the evolutive path of a given mass by induced mixing and other effects (not only photometric and geometric ones), the situation gets worse. The influence of the semi-empirical calibration of the colour index $\times M_{v}$ is another source of uncertainty in the process of comparison, so the high accuracy is surprising in the age determination of some clusters. We have tried to overcome some of these difficulties using the same set of models, incorporating new nuclear rates, and considering the geometric and photometric effect of rotation on the ages, as described by Pérez Hernández et al. (1999) and Claret \& Pérez Hernández (2007) (see Fig. 1 by Claret 2005b). However, in these calculations we do not consider the effects of either the induced mixing or the interaction of the meriodional circulation and shear turburlence on the evolutionary tracks. We expect that, if these effects are considered together with the mentioned geometric and photometric changes, the inferred ages will be even more different from those derived using standard (without rotation) isochrones.
2) A comparison of the ages of the systems directly with the time scales is not the best procedure. The differential equation for the orbital eccentricity must be integrated, including the PMS phase. The basic concepts used here to interpret the levels of synchronization and circularization have already been described in a previous paper on the subject (Claret \& Cunha 1997). Briefly, we integrated the differential equations that govern the eccentricity and axial rotation evolution for a given binary system until they decay to 0.05 per cent of their initial values. The time for which this occurs is named circularization (synchronization) time and the related $\log g$ is denoted by $\log g_{\text {cri }}$. We insist on these definitions in order to differentiate them from the classical time scale's meaning. For convective envelopes we adopted the formalism by Zahn (1989) instead of older formulae that consider the apsidal-motion constant $k_{2}$. Some numerical experiments were performed in order to compare the predictions based on both alternatives with similar results, although due to the differences between the tidal coefficient and the apsidal motion constant (see Fig. 3 by Claret \& Cunha 1997), the last version is a little bit more efficient.

We integrate the equations - adopting the tidal-torque and radiative damping mechanisms - but without taking the PMS phase into account. This is a limitation, but it represents a step forward since such an integration is not performed in most of the papers on circularization and only time scales are used (Fig. 8). The integration of the eccentricity equation considering PMS models implies some uncertainties: during our peliminary numerical tests we found that, for smaller periods, the radii of the stars are larger than their separation. In these cases it is assumed that the stars fill their respective Roche lobes. This seems to be a reasonable assumption but it does not guarantee the conservation of the total angular momentum, which is one of the fundamentals of the tidal evolution theory.

3) One should remember under which conditions the differential equations are derived (small eccentricity and little departure from the synchronism) and also consider the consequences of such an approach. As the initial eccentricity is unknown, only relative integrations are possible and we should keep the mentioned limitations in mind.

4) Due to the high dependency of the time scales on masses, relative radii, and orbital periods, the masses and radii for which the differential equations are integrated must be known better, otherwise serious problems of interpretations may appear (see the effect of changing the mass in Fig. 8).

5) A simple but very important question concerns the definition of the threshold period. There are some alternatives and counter-examples: a) the longest circular period? However, a binary system may be born with a very small eccentricity orbit. b) The shortest eccentric period? However a binary may be eccentric due to the presence of a third body, for example. If we select the first alternative, the tidal-torque braking is clearly not efficient enough to explain the observational aspects of Fig. 8. However, if the second option is used, the situation changes. In our opinion, the solution may lie somewhere between the two, since sharp definitions do not necessarily imply unbiased results. As the eccentricity-period distribution of clusters is often cloudy, we apply the least-square method to the observational $e-P$ diagrams in order to derive the critical period $P_{\text {critical }}$ (Fig. 8), instead of selecting extreme values (see Claret 2005b). Meibom \& Mathieu (2005) offer, in an independent way, a similar definition: the transition period. However, this procedure has no physical justification (p. 974) and makes use of an additional free parameter. The importance of such a new definition - no matter the selected method - is that all stars in the sample will 


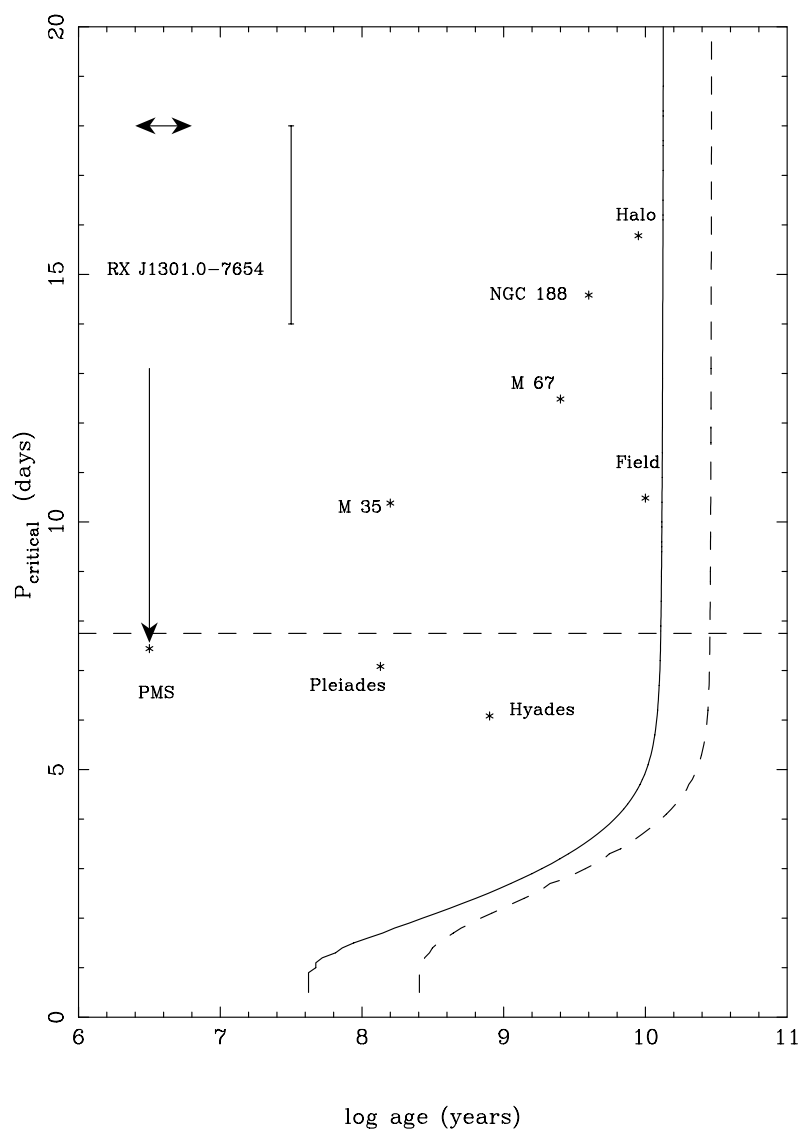

Fig. 8. Semi-empirical age versus the $P_{\text {critical }}$ diagram and the theoretical predictions. We have computed the critical times of circularization using the same grid of stellar models used to derive the ages of the clusters. The continuous line denotes the theoretical prediction for a $1 M_{\odot}$, while dashed-dotted line represents a $0.8 M_{\odot}$. The horizontal dashed line represents the prediction by Zahn \& Bouchet (1989) for PMS stars. The double arrow in the upper left corner represents the average uncertainties in the reddening and in the stellar models, while the vertical error bar represents the typical error for $P_{\text {critical }}$. The vertical arrow indicates the uncertainty on the period for PMS stars if the traditional definition $P_{\text {cut-off }}$ is used. Note that the position of Hyades is very different from that by Meibom \& Mathieu (2005).

influence the definition of the critical period and not only the two extreme values, as indicated above. For example, the position of RX J1301.0-7654 is crucial for determining the traditional cutoff period of PMS stars, as defined by Duquennoy et al. (1992) (the longest period for a circular orbit). In fact, the situation of this system is not clear concerning its definitive orbital elements, as far as we know. The question is still open but we think that the new definition, no matter the selected function, is a step forward towards better understanding the tidal evolution. If we consider the new definition of the critical period, some caution should be taken concerning its calculation. For example, if the least-square method is used to minimize the residuals, the distribution and the number of observational points over the plane $\log P-e$ should have some influence on $P_{\text {critical }}$ due to the nature of the leastsquare method. This could be reduced if the observations would be conveniently weighted.

6) The presence of a third body may alter the orbital behaviour of a given binary. The presence of a third body in a binary system belonging to a cluster is more probable than in the case of isolated binaries.
7) There are still very large uncertainties in the theory of the transport of energy by convection, and the derivation of the differential equations governing the eccentricity (and the rotational velocity) must be affected by this unaccuracy. As is known, we do not have an acceptable theory of convection applicable to stellar interiors. The corresponding error bar is still hard to establish and is not displayed in Fig. 8. However, we can indirectly asses the role of the convective envelope's depth in the tidal-breaking mechanism's efficiency. To do that, we changed the integration conditions of the parameter $\lambda_{2}$ (see Claret \& Cunha 1997). Even considering the lower limit of that integration equal to zero, the efficiency does not increase very much (see Fig. 9a). Although the role of convection in stellar evolution is much more complex than our numerical experiment suggests, the present result may indicate that it is not the only cause of the global disagreement between theory and observations.

8) To evaluate the influence of the intrinsic uncertainties of stellar models on the theoretical circularization times, we carried out some calculations by assuming symmetrical errors in the radius of about $20 \%$. The results are displayed in Fig. 9b. It seems, at first glance, that such an uncertainty on the radius is, perhaps, too large, although this difference is perfectly acceptable depending on the metallicity of the models, for a given age. In fact, for more evolved models, it may be larger. However, the dashed lines in Fig. 9b clearly indicate that these models cannot explain the disagreement; only with an uncertainty of $250 \%$ in radius, which clearly contradicts the actual comparison between double-lined binaries and evolutionary-model predictions, could fit the observations. In spite of that, these calculations are useful for evaluating the effects of the radius accuracy on the levels of circularization. On the other hand, the present grids can be used to evaluate the effects of a change in metallicity on the efficiency of the tidal-breaking mechanism (Fig. 9c). Even considering the mentioned theoretical error bars, the tidal-breaking mechanism is not efficient enough to explain the observed age- $P_{\text {critical }}$ diagram.

\subsection{The circularization times in isolated close binaries}

Isolated close binaries stars present, mainly if they are doublelined eclipsing binaries, a better determination of their absolute dimensions than those belonging to clusters. At first glance, this would reduce one of the problems previously pointed out (accuracy of absolute dimensions). However, as shown by Claret \& Cunha (1997), the radiative damping and the tidal-torque mechanisms are not efficient for fitting the observed eccentricities of a sample of about 40 DLEB: they cannot explain some systems with circular orbits that present smaller ages than their respective critical times, unless they were born with zero eccentricities. A numerical experiment indicates that only the introduction of an artificial enhancement (about two orders of magnitude) of such process it makes possible to fit the data.

As we have seen, the better knowlegde of the absolute dimensions does not guarantee good agreement with observations. This, however, does not mean that point four in the previous section loses importance. The improvement in the determination of the masses (and radii, if possible) is critical for a comparison with the times of circularization. Depending on the mass range, both processes (tidal-torque and radiative damping) may be acting simultaneously, increasing the efficiency of circularization.

Since our paper on the circularization levels of double-lined eclipsing binaries (Claret \& Cunha 1997), several papers on the absolute dimensions determination have been published by, among others, Lacy and collaborators (see the reference list). In 

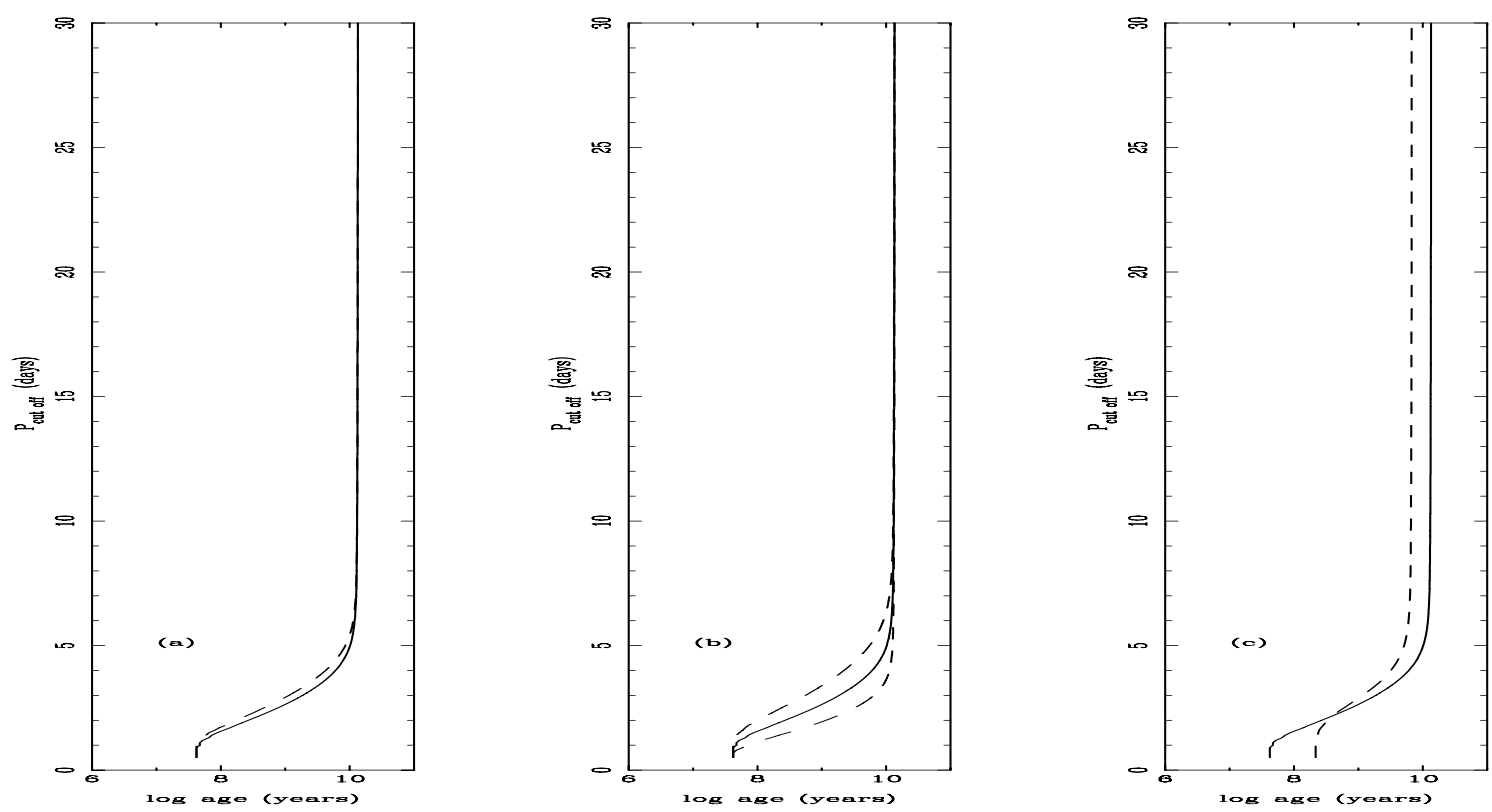

Fig. 9. Effect of changing the depth of the convective envelope $x_{\mathrm{bf}} \mathbf{a}$ ), of changing the radius $\mathbf{b}$ ), and of changing the chemical composition $\mathbf{c}$ ). a) The continuous line represents the standard calculation for a $1 M_{\odot}$ model with true values of $x_{\mathrm{bf}}$, while the dashed one denotes the calculation by assuming $x_{\mathrm{bf}}=0$ during the evolution of such model. b) The continuous line represents the standard calculation for a $1 M_{\odot}$ model. The two dashed lines denote the calculation carried out by assuming an increase (decrease) of $20 \%$ in the radius. c) The continuous line represents the standard calculation for a $1 M_{\odot}$ model (with $Z=0.02$ ), while the dashed line denotes the same model but with $Z=0.10$.

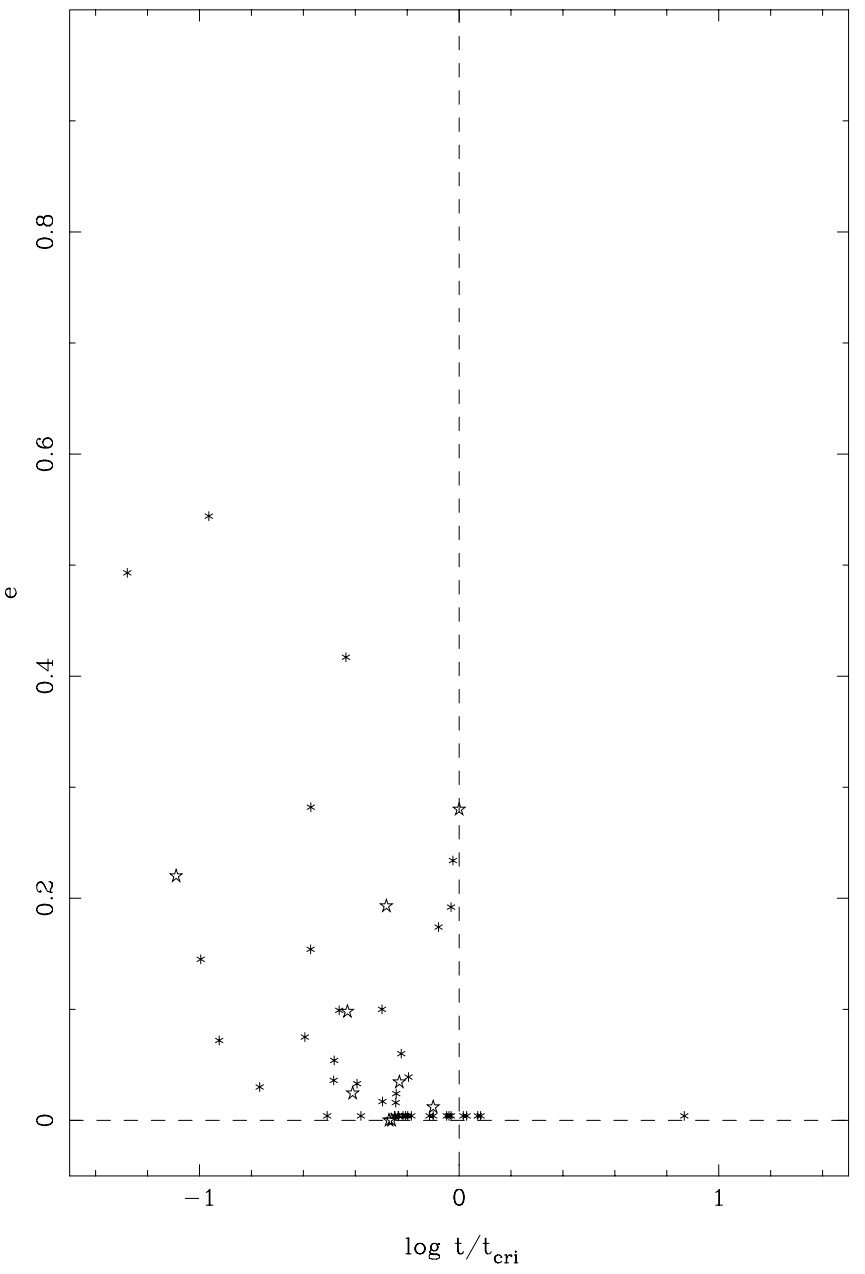

Fig. 10. Eccentricity as a function of the age and the critical time for circularization. New data of double-lined eclipsing binary systems were added and are plotted as open stars. order to increase our sample of double-lined eclipsing binaries, we added 10 new systems to our 1997 sample (Fig. 10). These new data do not change the general trend of the diagnostic diagram $e-\log \left(\frac{t}{t_{\mathrm{cri}}}\right)$. Obviously, much more systems are needed to draw a more definitive conclusion, but the aspect of Fig. 10 seems to reinforce the low efficiency of the tidal-torque and radiative damping mechanisms.

As a final remark, we would like to insist that the calculation of circularization times should start from the PMS stage but should not only consider such a stage; later evolutionary phases should be also considered. As already pointed out, the PMS integration may lead to problems with angular momentum conservation for shorter periods. We are now working on this subject and expect to present the results in the near future.

\section{Tables}

The present models (90 tables) can be retrieved from the CDS via anonymous ftp. The available data for each model are the age, $\log L, \log g, \log T_{\text {eff }}$, the mass, the $\log$ arithm of the massloss rate $\left(M_{\odot} /\right.$ year $)$, the logarithm of the central temperature, the logarithm of the central density, the core $q_{c}$ in fraction of the total mass of the star, the central and surface abundances of $X$, $Y,{ }^{12} \mathrm{C},{ }^{13} \mathrm{C},{ }^{14} \mathrm{~N},{ }^{16} \mathrm{O},{ }^{17} \mathrm{O},{ }^{18} \mathrm{O},{ }^{20} \mathrm{Ne},{ }^{22} \mathrm{Ne},{ }^{24} \mathrm{Mg},{ }^{25} \mathrm{Mg}$, and ${ }^{26} \mathrm{Mg}, \log k_{2}, \log k_{3}, \log k_{4}, x_{\mathrm{bf}}, \log E_{2}, E, \alpha_{P}, \beta$ and $\beta_{1}$ (for more details, see Paper I).

Acknowledgements. I am in debt to L. F. Miranda and N. Mowlavi for their comments and suggestions. The Spanish MEC (AYA2006-06375) is gratefully acknowledged for its support during the development of this work. Use was made of the WEBDA database, operated at the University of Vienna, Austria.

\section{References}

Alexander, D. R., \& Ferguson, J. W. 1994, ApJ, 437, 879

Baldwin, J. A., Hamann, F., Korista, K. T., et al. 2003, ApJ, 583, 649

Carraro, G., Villanova, S., Demarque, P., et al. 2006, ApJ, 643, 1151 
Claret, A. 1998, A\&AS, 131, 395

Claret, A. 2000, A\&A, 259, 289

Claret, A. 2004, A\&A, 424, 919 (Paper I)

Claret, A. 2005a, A\&A, 440, 647

Claret, A. 2005b, in Tidal evolution and oscillations in binary stars, ed. A. Claret,

A. Giménez \& J.-P. Zahn, ASP Ser., 333, 122

Claret, A. 2006, A\&A, 453, 769

Claret, A., \& Cunha, N. C. S. 1997, A\&A, 318, 187

Claret, A., \& Pérez Hernández, F. 2007, in preparation

Claret, A., Giménez, A., \& Cunha, N. C. S. 1995, A\&A, 299, 724

Duquennoy, A., Mayor, M., \& Mermilliod, J.-C. 1992, in Binaries as tracers of

stellar formation, ed. Duquennoy \& Mayor (Cambridge University Press), 52

Formicola, A., Imbriani, G.; Costantini, H., et al. 2004, Phys. Lett. B, 591, 61

Gratton, R., Bragaglia, A., Carreta, E., \& Tosi, M. 2006, ApJ, 642, 462

Iglesias, C. A., \& Rogers, F. J. 1996, ApJ, 464, 943

Itoh, N., Adachi, T., Nakagawa, M., Kohyama, Y., \& Munakata, H. 1989, ApJ, 339,354

Korista, K., Hamman, F., Fergunson, J., \& Ferland, G. 1996, ApJ, 461, 641

Lacy, C. H. S., Torres, G., Claret, A., \& Sabby, J. A. 2000a, AJ, 120, 3226

Lacy, C. H. S., Torres, G., Claret, A., et al. 2000b, AJ, 119, 1389

Lacy, C. H. S., Torres, G., Claret, A., \& Sabby, J. A. 2002, AJ, 123, 1013

Lacy, C. H. S., Vaz, L. P. R., Claret, A., \& Sabby, J. A. 2003, AJ, 128, 1324

Lacy, C. H. S., Torres, G., Claret, A., et al. 2004a, AJ, 128, 3005
Lacy, C. H. S., Torres, G., Claret, A., \& Sabby, J. A. 2004b, AJ, 128, 1840 Lacy, C. H. S., Torres, G., Claret, A., \& Sabby, J. A. 2004c, AJ, 128, 1340 Lacy, C. H. S., Torres, G., Claret, A., \& Vaz, L. P. R. 2005, AJ, 130, 2838 Mayor, M., \& Mermilliod, J. C. 1984, IAU 115, 411

Meynet, G. 1998, ASPC, 192, 41,

Meibom, S., \& Mathiew, R. D. 2005, ApJ, 620, 970

Michalska, G., \& Pigulski, A. 2005, A\&A, 434, 89

Moehler, S., \& Sweigart, A. V. 2006, A\&A, 455, 943

Mowlavi, N., Schaerer, D., Meynet, G., et al. 1998, A\&AS, 128, 471

Nugis, T., \& Lamers, H. J. G. L. M. 2000, A\&A, 360, 227

Pérez Hernández, F., Claret, A., Hernández, M. M., \& Michel, E. 1999, A\&A, 356, 586

Rattenbury, N. J., Abe, F., Bennett, D. P., et al. 2005, A\&A, 439, 645

Runkle, R. C. 2003, Ph. D. Thesis, University of North Carollina, unpublished

Sadler, E. M. 1992, in Metal-Rich Stars in the Galactic Bulge and Their Implications for Elliptical Galaxies, ed. B., \& A. Renzini, 149, 41

Southworth, J. Smalley, B., Maxted, P. F. L., Claret, A., \& Etzel, P. B. 2005, MNRAS, 363, 529

Tassoul, J. L. 1995, ApJ, 444, 338

Tassoul, J. L., \& Tassoul, M. 1996, Fund. of Cosmic Phys., 16, 337

Twarog, B. A., Twarog, B. J. A., \& De Lee, N. 2003, AJ, 125, 1383

Zahn, J-P. 1977, A\&A, 57, 383

Zahn, J-P. 1989, A\&A, 220, 112 